\title{
Reação de genótipos de soja ao crestamento foliar de cercospora
}

\author{
Angela Sathiko Kudo ( $\left.{ }^{1}\right)$; Luiz Eduardo Bassay Blum (2*) \\ (') Conselho Nacional de Desenvolvimento Científico e Tecnológico (CNPq), SEPN 509, 3.andar, 70750-501 Brasília (DF). \\ (2) Universidade de Brasília, Departamento de Fitopatologia, Campus Universitário Darcy Ribeiro, 70910-900 Brasília (DF). \\ (*) Autor correspondente: luizblum@unb.br
}

Recebido: 25/mai./2009; Aceito: 30/out./2010.

\begin{abstract}
Resumo
Foram realizados dois experimentos em um delineamento em blocos ao acaso com três repetições, em Cristalina (GO), na safra de 2005/2006, para avaliar a reação de genótipos de soja convencional e transgênica (RR) ao crestamento foliar causado por Cercospora kikuchii. No experimento 1, foram avaliados 86 genótipos de soja convencional e no experimento 2, 30 genótipos de soja convencional e transgênica quanto à reação ao crestamento foliar. Como padrões de genótipos convencionais foram usados Emgopa 313 e Msoy 8001 e como genótipos transgênicos Msoy 8585 RR e Msoy 8787 RR. Os genótipos com menor incidência (<1\%) da doença no experimento 1 foram: GT04-7229, GT04-8987, GT04-8902, GT04-7991, GT04-8218, GT04-9501, GT04-7020, GT04-7505 e GT04-7439. No experimento 2, os menos afetados foram: GT04-7437 RR, GT049192 RR, GT04-8091 RR, GT04-8203, GT04-9265 RR e GT04-9085 RR. Não houve diferença na intensidade de doença entre genótipos transgênicos e convencionais. A intensidade do crestamento nos experimentos foi baixa (área foliar lesionada de 0 a 1,5\% e incidência de 0 a 20\%).
\end{abstract}

Palavras-chave: Cercospora kikuchii, Glycine max (L.) Merr., soja transgênica.

\section{Reaction of soybean genotypes to cercospora leaf blight}

\section{Abstract}

This study evaluated the reaction of soybean genotypes (conventional and transgenic) to cercospora leaf blight (Cercospora kikuchii). Two experiments in a completely randomized block design with three replications were carried out (Cristalina GO and Brasília DF, Brazil) during the crop period of 2005/2006. In the experiment 1, 86 non-transgenic genotypes were evaluated, while in the experiment 2, 30 transgenic (RR) and non-transgenic genotypes were tested. For comparisons purposes the following genotypes were used: conventional Emgopa 313 and Msoy 8001, and transgenic Msoy 8585 RR and Msoy 8787 RR. The genotypes with lower levels of disease incidence (<1\%) were: experiment 1 - GT04-7229, GT04-8987, GT04-8902, GT04-7991, GT04-8218, GT04-9501, GT04-7020, GT04-7505 and GT04-7439; experiment 2 - GT04-7437 RR, GT04-9192 RR, GT04-8091 RR, GT04-8203, GT04-9265 RR and GT04-9085 RR. There was no significant difference in disease intensity between transgenic and non-transgenic genotypes and the Cercospora leaf blight intensity reported in this study was low when considering lesions on leaf area $(<1.5 \%)$ and disease incidence $(<20 \%)$.

Key words: Cercospora kikuchii, Glycine max (L.) Merr., transgenic soybean.

A importância do crestamento foliar de cercospora [Cercospora kikuchii (Матsumoto e TOMOYasu) Gardner] em soja [Glycine max (L.) Merr.] é indiscutível (Wrather et al., 1997), embora nos últimos anos a ferrugem asiática venha se destacando (Phakopsora pachyrhizi H. Sydow e Sydow) (Embrapa, 2008). No Brasil, a doença está disseminada por todas as regiôes produtoras de soja, porém com ocorrência mais séria nas regiōes mais quentes e chuvosas dos cerrados (Almeida et al., 1997).

Entre as principais medidas de controle do crestamento foliar estáo a utilizaçáo de sementes sadias, o tratamento de sementes, a incorporaçáo de restos culturais, a aplicação de fungicidas entre o florescimento e o enchimento de grãos e a rotação com espécies de plantas não suscetíveis (Sinclair e BaCKman, 1989; EMBRAPA, 1999; 2008).

O controle da doença pode ser conseguido por meio da resistência genética, apesar de ainda não ter sido identificado gene de resistência a C. kikuchii (Almeida et al., 2005), sendo o modo mais eficaz e econômico de controle de doenças (ЕмBrapa, 2008; Juliatti et al., 2006). Além disso, de acordo com diversos autores, o fungo possui alta variabilidade genética (Walters, 1978; Almeida et al., 2001, 2005), o que leva à necessidade de constante busca por fontes de resistência. Assim, o objetivo deste estudo foi avaliar 
a reação de genótipos de soja ao crestamento foliar sob condiçôes de campo.

Foram realizados dois experimentos de campo durante a safra 2005/2006 com o objetivo de avaliar a reação ao crestamento foliar de Cercospora de genótipos de soja convencional e transgênica (RR: 'Roundup Ready'). A cada safra foram selecionados os genótipos com melhor desempenho levando-se em consideração não só os níveis de reação a doenças [Ferrugem asiática (Phakopsora pachyrhizi), cercosporioses (Cercospora sojina e C. kikuchii), antracnose (Colletotrichum truncatum), podridão vermelha (Fusarium solani), mancha parda (Septoria glycines) e oídio (Erysiphe diffusa)], mas também fatores de interesse agronômico e comercial.

Os experimentos foram desenvolvidos em Cristalina (GO). Todas as parcelas desses experimentos foram adubadas com $230 \mathrm{~kg} \mathrm{ha}^{-1}$ de NPK 5-36-00 + micronutrientes, e em cada parcela havia seis linhas de $6 \mathrm{~m}$ de comprimento, espaçadas de $0,45 \mathrm{~m}$ e com 15 plantas $\mathrm{m}^{-1}$. As avaliaçôes foram bissemanais e as amostragens feitas coletando-se seis trifolíolos (18 folíolos) da parte média das plantas de cada parcela, e após acondicionados em sacos plásticos e levados ao laboratório.

A semeadura em ambos os experimentos foi realizada em 15/11/05. O delineamento experimental foi o de blocos casualizados com três repetiçôes. No experimento 1 , foram avaliados 86 genótipos de soja convencional (Tabela 1) e no experimento 2,30 genótipos de soja convencional e transgênica (Tabela 2) quanto à reação ao crestamento foliar. Nos experimentos foram utilizados como padróes de genótipos convencionais o Emgopa 313 e Msoy 8001 e como padróes de genótipos transgênicos o Msoy 8585 RR e Msoy 8787 RR. Foram aplicados os seguintes defensivos: (a) 15/12/05 [fungicida: Epoxiconazole + Piraclostrobina (Opera) na dose comercial de $0,5 \mathrm{~L} \mathrm{ha}^{-1}$ - para controle da ferrugem asiática (Phakopsora pachyrhizi); inseticida/ acaricida: Metamidofós na dose comercial de $\left.0,8 \mathrm{~L} \mathrm{ha}^{-1}\right] \mathrm{e}$; (b) 5/1/06 [Azoxistrobina + Ciproconazole (Priori Xtra) na dose $0,3 \mathrm{~L} \mathrm{ha}^{-1}$; Metamidofós na dose de $0,8 \mathrm{~L} \mathrm{ha}^{-1}$.

Foram avaliadas a severidade [estimativa da porcentagem de área foliar lesionada (AFL)] e a incidência da doença nos folíolos [porcentagem (IDF)]. As avaliaçôes ocorreram em $8 / 3$ e em 22/3/06. Para a calibragem visual da avaliação de AFL, utilizou-se a escala diagramática publicada por MarTins et al. (2004), para quantificação do crestamento por C. kukuchii e da mancha parada por S. glycines.

As análises estatísticas foram feitas com auxílio do programa SAS [SAS Institute Inc. (1999)] e Sisvar [Versão 5.1 (2007)]. Os dados foram transformados em $(X+0,5)^{1 / 2}$, conforme indicado por Steel e Torrie (1980). Foi realizada a análise de variância e as médias foram comparadas pelo teste de Tukey ao nível de 5\% de probabilidade de erro. Foram feitas análises de correlação linear entre as variáveis avaliadas baseando-se na significância de seus coeficientes. Para a classificação da intensi- dade da correlação ( $\mathrm{p} \leq 0,01)$ (Guerra e Livera, 1999), foi considerada muito forte $(\mathrm{r}=0,91$ a 1,00$)$, forte $(\mathrm{r}=$ $0,71$ a 0,90$)$, média $(r=0,51$ a 0,70$)$ e fraca $(r=0,31$ a $0,50)$.

No experimento 1, as avaliaçôes diferiram entre si para a variável $A F L$, e na primeira avaliação observaram-se os maiores níveis de doença. Para a variável IDF não houve diferença entre as avaliaçóes, com médias de 5,23\% e $4,24 \%$ em R5 e R7 respectivamente. A menor incidência no estágio R7 foi devido à desfolha. No experimento 2 , houve diferença entre as épocas de avaliação para a variável IDF, sendo a primeira avaliaçáo o valor de incidência de $2,45 \%$ e a segunda com $6,82 \%$.

Houve diferença entre genótipos quanto à IDF no experimento 1 (Tabela 1). Nos genótipos GT04-7229, GT04-8987, GT04-8902, GT04-7991 e GT04-8218 não se notaram sintomas visíveis de crestamento, mas diferiram do genótipo GT04-9060, em que houve maior incidência $(-20 \%)$. No entanto, nenhum dos genótipos supracitados diferiu de Emgopa 313 e Msoy 8001.

No experimento 2 (Tabela 2), houve diferença entre genótipos para a variável IDF. No genótipo GT04-7437 RR não foram notados sintomas visíveis de crestamento foliar, diferindo significativamente de GT04-9051, que foi o genótipo com maior incidência da doença $(-16,5 \%)$. No entanto, nenhum dos genótipos citados diferiu das cultivares Msoy 8585 RR e Msoy 8787 RR .

Em ambos os experimentos, não houve diferença entre genótipos quanto à AFL. Os genótipos com mais doença foram GT04-7629 e GT04-9180 no experimento 1. Já no experimento 2, no genótipo GT04-7437 RR não ocorreu doença. Os genótipos com maior severidade de crestamento foliar foram GT04-8202 e GT04-9051.

A variação da AFL entre genótipos foi de 0 a 1,5\% no experimento 1 e de 0 a $0,28 \%$ no experimento 2 . No experimento 2, nos genótipos transgênicos (RR), verificaram-se menores níveis de doença que nos genótipos convencionais para as variáveis IDF e AFL. Houve correlação positiva forte entre as variáveis INC e AFL nos experimentos $1(\mathrm{r}=0,74)$ e $2(\mathrm{r}=0,72)$.

Nos experimentos 1 e 2 foram feitas duas quantificaçóes de doença no fim do ciclo da cultura. Os trabalhos realizados por outros autores (Hepperly, 1984; Miller e Roy, 1982, Roy e Abney, 1976; Wilcox e AbNey, 1973) envolvendo C. kikuchii têm sido concentrados principalmente na infecção em vagem ou em folha no fim do ciclo.

Godoy e CANTeri (2004) relataram que esta doença foi observada a partir de 120 dias após a semeadura no estágio R6 (vagens com sementes verdes que preenchem totalmente a cavidade) (Fehr e Caviness, 1981). Orth e Sснин (1994) relataram que os sintomas típicos de crestamento foliar nos folíolos não foram discernidos antes do estágio R5 nas cultivares estudadas. Assim, esses autores avaliaram a severidade por meio da AFL no estágio R6 das plantas, no qual foram observadas pequenas manchas 
Tabela 1. Reação de genótipos de soja ao crestamento foliar (Cercospora kikuchii) em experimento desenvolvido em Cristalina (GO), na safra 2005/2006

\begin{tabular}{|c|c|c|c|c|c|c|c|c|c|}
\hline Genótipo & AFL & & IDF & & Genótipo & AFL & & IDF & \\
\hline GT04-7229 & 0 & a & 0 & $b$ & Emgopa 313 & 0,235 & a & 4,190 & $a b$ \\
\hline GT04-8987 & 0 & $\mathrm{a}$ & 0 & $b$ & GT04-9506 & 0,835 & $\mathrm{a}$ & 4,557 & $a b$ \\
\hline GT04-8902 & 0 & $\mathrm{a}$ & 0 & $\mathrm{~b}$ & GT04-9144 & 0,150 & $\mathrm{a}$ & 4,716 & $a b$ \\
\hline GT04-7991 & 0 & $\mathrm{a}$ & 0 & $b$ & GT04-8908 & 0,182 & $\mathrm{a}$ & 4,795 & $a b$ \\
\hline GT04-8218 & 0 & a & 0 & $b$ & GT04-8045 & 0,325 & a & 4,856 & $a b$ \\
\hline GT04-9501 & 0,035 & $\mathrm{a}$ & 0,284 & $a b$ & GT04-9133 & 0,153 & $\mathrm{a}$ & 5,021 & $a b$ \\
\hline GT04-7020 & 0,009 & $\mathrm{a}$ & 0,499 & $a b$ & GT04-7722 & 0,554 & a & 5,034 & $a b$ \\
\hline GT04-7505 & 0,018 & $\mathrm{a}$ & 0,839 & $a b$ & GT04-7622 & 0,153 & $\mathrm{a}$ & 5,117 & $a b$ \\
\hline GT04-7439 & 0,034 & $\mathrm{a}$ & 0,839 & $a b$ & GT04-8777 & 0,228 & a & 5,291 & $a b$ \\
\hline GT04-7006 & 0,016 & $\mathrm{a}$ & 1,138 & $a b$ & GT04-9529 & 0,414 & $\mathrm{a}$ & 5,291 & $a b$ \\
\hline GT04-8326 & 0,018 & $\mathrm{a}$ & 1,168 & $a b$ & GT04-7495 & 0,374 & a & 5,349 & $a b$ \\
\hline GT04-8114 & 0,027 & $\mathrm{a}$ & 1,168 & $a b$ & GT04-9500 & 0,375 & a & 5,439 & $a b$ \\
\hline GT04-7247 & 0,035 & $\mathrm{a}$ & 1,168 & $a b$ & GT04-7988 & 0,175 & a & 5,475 & $a b$ \\
\hline GT04-7019 & 0,086 & $\mathrm{a}$ & 1,168 & $a b$ & GT04-8989 & 0,081 & a & 5,612 & $a b$ \\
\hline GT04-7724 & 0,218 & $\mathrm{a}$ & 1,601 & $a b$ & GT04-9187 & 0,441 & $\mathrm{a}$ & 6,225 & $a b$ \\
\hline GT04-8802 & 0,078 & $\mathrm{a}$ & 1,677 & $a b$ & GT04-7424 & 0,165 & $\mathrm{a}$ & 6,233 & $a b$ \\
\hline GT04-9521 & 0,060 & $\mathrm{a}$ & 1,971 & $a b$ & GT04-9182 & 0,432 & $\mathrm{a}$ & 6,233 & $a b$ \\
\hline GT04-7781 & 0,068 & a & 1,971 & $a b$ & GT04-9162 & 1,309 & $a$ & 6,949 & $a b$ \\
\hline GT04-8101 & 0,077 & $\mathrm{a}$ & 1,971 & $a b$ & GT04-9189 & 0,131 & $\mathrm{a}$ & 7,142 & $a b$ \\
\hline GT04-7071 & 0,045 & a & 2,009 & $a b$ & GT04-7470 & 1,279 & $a$ & 7,593 & $a b$ \\
\hline GT04-7615 & 0,053 & a & 2,009 & $a b$ & GT04-9217 & 0,388 & a & 8,478 & $a b$ \\
\hline GT04-9141 & 0,240 & $\mathrm{a}$ & 2,072 & $a b$ & GT04-7253 & 0,931 & $a$ & 8,798 & $a b$ \\
\hline GT04-8012 & 0,073 & $\mathrm{a}$ & 2,083 & $a b$ & GT04-8999 & 0,449 & $\mathrm{a}$ & 9,004 & $a b$ \\
\hline GT04-8276 & 0,092 & $\mathrm{a}$ & 2,083 & $a b$ & GT04-7677 & 0,562 & $\mathrm{a}$ & 9,112 & $a b$ \\
\hline GT04-8093 & 0,059 & a & 2,309 & $a b$ & GT04-7605 & 1,004 & a & 9,112 & $a b$ \\
\hline GT04-9143 & 0,069 & $\mathrm{a}$ & 2,493 & $a b$ & Msoy 8001 & 0,800 & $\mathrm{a}$ & 9,490 & $a b$ \\
\hline GT04-8356 & 0,054 & $a$ & 2,534 & $a b$ & GT04-7689 & 0,690 & a & 10,115 & $a b$ \\
\hline GT04-7765 & 0,062 & $\mathrm{a}$ & 2,534 & $a b$ & GT04-9516 & 0,358 & $\mathrm{a}$ & 10,192 & $a b$ \\
\hline GT04-8524 & 0,070 & $\mathrm{a}$ & 2,534 & $a b$ & GT04-8423 & 0,534 & a & 10,287 & $a b$ \\
\hline GT04-7626 & 0,062 & $\mathrm{a}$ & 2,549 & $a b$ & GT04-7310 & 0,902 & $a$ & 10,589 & $a b$ \\
\hline GT04-9533 & 0,063 & $\mathrm{a}$ & 2,976 & $a b$ & GT04-8270 & 0,166 & a & 11,101 & $a b$ \\
\hline GT04-7823 & 0,070 & a & 2,976 & $a b$ & GT04-8086 & 1,189 & $a$ & 11,521 & $a b$ \\
\hline GT04-8254 & 0,111 & $\mathrm{a}$ & 2,990 & $a b$ & GT04-9078 & 1,260 & $\mathrm{a}$ & 12,023 & $a b$ \\
\hline GT04-8796 & 0,054 & $\mathrm{a}$ & 3,020 & $a b$ & GT04-9196 & 0,548 & $\mathrm{a}$ & 12,096 & $a b$ \\
\hline GT04-9009 & 0,227 & $\mathrm{a}$ & 3,094 & $a b$ & GT04-7100 & 0,437 & a & 12,238 & $a b$ \\
\hline GT04-9065 & 0,079 & $\mathrm{a}$ & 3,108 & $a b$ & GT04-7606 & 0,552 & $a$ & 12,323 & $a b$ \\
\hline GT04-9514 & 0,080 & $\mathrm{a}$ & 3,108 & $a b$ & GT04-8990 & 0,800 & $\mathrm{a}$ & 13,954 & $a b$ \\
\hline GT04-9526 & 0,060 & $\mathrm{a}$ & 3,208 & $a b$ & GT04-8116 & 0,863 & $a$ & 14,809 & $a b$ \\
\hline GT04-7379 & 0,321 & $\mathrm{a}$ & 3,589 & $a b$ & GT04-7857 & 0,769 & $\mathrm{a}$ & 15,101 & $a b$ \\
\hline GT04-9127 & 0,063 & $\mathrm{a}$ & 3,637 & $a b$ & GT04-9100 & 1,062 & $\mathrm{a}$ & 16,499 & $a b$ \\
\hline GT04-7536 & 0,332 & a & 4,043 & $a b$ & GT04-7629 & 1,500 & a & 16,980 & $a b$ \\
\hline GT04-7621 & 0,396 & $\mathrm{a}$ & 4,043 & $a b$ & GT04-9180 & 1,435 & $\mathrm{a}$ & 18,877 & $a b$ \\
\hline GT04-7671 & 0,437 & $\mathrm{a}$ & 4,151 & $a b$ & GT04-9060 & 0,976 & a & 20,032 & $\mathrm{a}$ \\
\hline
\end{tabular}

AFL (área foliar lesionada, \%); IDF (\% incidência); Valores seguidos pela mesma letra não diferem entre si pelo teste de Tukey ao nível de $5 \%$ de probabilidade. Coeficiente de variação: $\mathrm{CV}(\mathrm{AFL})=35,7 \% ; \mathrm{CV}(\mathrm{IDF})=67,3 \%$.

da doença. Diferente dos dois trabalhos supracitados, nestes dois experimentos a primeira avaliação foi realizada no estágio R5, na qual já foram observados sintomas de crestamento foliar, embora com menor severidade.

Nos experimentos 1 e 2 a variação de INC na avaliação em R5 (2,5\% e 5,2\%) foi próxima ao valor obtido por KuINGelfuss e Yorinori (2001) na avaliação no estádio R5.4 (2,5\%). No entanto, no estádio R7 houve variação de $4,2 \%$ a $6,8 \%$ nos experimentos 1 e 2 , resultados muito diferentes do obtido pelos autores, os quais observaram que a incidência teve média de $84,2 \%$ na fase R7.2.

No trabalho realizado por OrTH e SchUH (1994), no qual foram analisadas 17 cultivares de soja com relação à resistência ao crestamento foliar, não houve diferença entre os genótipos. Entretanto, os níveis de severidade

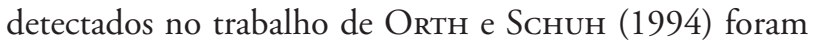
maiores, com variação de $2,2 \%$ a $10,5 \%$ para a AFL, uma vez que nos experimentos supracitados a variação da $\mathrm{AFL}$ 
Tabela 2. Reação de genótipos de soja transgênicos (RR: 'Roundup Ready') e convencionais ao crestamento foliar (Cercospora kikuchii) em experimento de campo desenvolvido em Cristalina (GO), na safra 2005/2006

\begin{tabular}{|lllll|}
\hline Genótipo & \multicolumn{2}{c}{ AFL } & \multicolumn{2}{c|}{ IDF } \\
\hline GT04-7437 RR & $0^{2}$ & $\mathrm{a}$ & 0 & $\mathrm{~b}$ \\
\hline GT04-9192 RR & 0,005 & $\mathrm{a}$ & 0,499 & $\mathrm{ab}$ \\
\hline GT04-8091 RR & 0,010 & $\mathrm{a}$ & 0,839 & $\mathrm{ab}$ \\
\hline GT04-8203 & 0,030 & $\mathrm{a}$ & 0,839 & $\mathrm{ab}$ \\
\hline GT04-9265 RR & 0,024 & $\mathrm{a}$ & 0,839 & $\mathrm{ab}$ \\
\hline GT04-9085 RR & 0,040 & $\mathrm{a}$ & 2,009 & $\mathrm{ab}$ \\
\hline GT04-8211 & 0,042 & $\mathrm{a}$ & 2,309 & $\mathrm{ab}$ \\
\hline GT04-9062 RR & 0,087 & $\mathrm{a}$ & 2,309 & $\mathrm{ab}$ \\
\hline GT04-8149 & 0,067 & $\mathrm{a}$ & 2,534 & $\mathrm{ab}$ \\
\hline GT04-9172 & 0,016 & $\mathrm{a}$ & 2,976 & $\mathrm{ab}$ \\
\hline GT04-8327 & 0,027 & $\mathrm{a}$ & 3,020 & $\mathrm{ab}$ \\
\hline GT04-7643 RR & 0,054 & $\mathrm{a}$ & 3,208 & $\mathrm{ab}$ \\
\hline GT04-8298 & 0,071 & $\mathrm{a}$ & 3,374 & $\mathrm{ab}$ \\
\hline GT04-8210 & 0,080 & $\mathrm{a}$ & 3,535 & $\mathrm{ab}$ \\
\hline GT04-9119 & 0,078 & $\mathrm{a}$ & 3,637 & $\mathrm{ab}$ \\
\hline GT04-9107 & 0,026 & $\mathrm{a}$ & 4,304 & $\mathrm{ab}$ \\
\hline Ms0y 8585RR & 0,052 & $\mathrm{a}$ & 4,795 & $\mathrm{ab}$ \\
\hline Msoy 8787RR & 0,081 & $\mathrm{a}$ & 5,520 & $\mathrm{ab}$ \\
\hline GT04-8319 RR & 0,114 & $\mathrm{a}$ & 5,671 & $\mathrm{ab}$ \\
\hline GT04-7447 & 0,091 & $\mathrm{a}$ & 5,801 & $\mathrm{ab}$ \\
\hline GT04-8077 & 0,129 & $\mathrm{a}$ & 6,495 & $\mathrm{ab}$ \\
\hline GT04-8290 & 0,197 & $\mathrm{a}$ & 6,784 & $\mathrm{ab}$ \\
\hline GT04-8150 & 0,170 & $\mathrm{a}$ & 7,287 & $\mathrm{ab}$ \\
\hline GT04-8212 & 0,090 & $\mathrm{a}$ & 8,125 & $\mathrm{ab}$ \\
\hline GT04-8242 & 0,116 & $\mathrm{a}$ & 8,478 & $\mathrm{ab}$ \\
\hline GT04-8279 & 0,221 & $\mathrm{a}$ & 10,171 & $\mathrm{ab}$ \\
\hline GT04-8202 & 0,283 & $\mathrm{a}$ & 12,182 & $\mathrm{ab}$ \\
\hline GT04-9102 & 0,274 & $\mathrm{a}$ & 16,477 & $\mathrm{a}$ \\
\hline GT04-9051 & $\mathrm{a}$ & 12,641 & $\mathrm{ab}$ \\
\hline AFL & & & & \\
\hline
\end{tabular}

AFL (área foliar lesionada, \%); IDF (\% incidência);Valores seguidos pela mesma letra náo diferem entre si pelo teste de Tukey ao nível de $5 \%$ de probabilidade. Coeficiente de variaçăo: $\mathrm{CV}(\mathrm{AFL})=11,8 \% ; \mathrm{CV}(\mathrm{IDF})=67,3 \%$.

foi de 0 a 1,5\%. Estas diferenças em resultados podem estar associadas ao local e aos genótipos testados.

Segundo Orth e Schum (1994), a seleção para resistência sob condiçốes de campo é influenciada por variações na pressão de doença e ambiente. Santos et al. (2008) verificaram que houve diferença nos níveis de cercosporiose do cafeeiro de uma safra para outra, e atribuíram tal diferença ao manejo referente à nutrição das plantas. No presente trabalho, considerando que ambas as lavouras de soja foram desenvolvidas sob mesmo tipo de solo, disponibilidade de água, cultivar, idades das plantas e adubação, as diferenças nos níveis de crestamento foliar possivelmente ocorreram devido aos fatores climáticos.

Sснин (1991) relatou que a temperatura ótima para a infecção das plantas por C. kikuchii foi de $25^{\circ} \mathrm{C}$; também o autor relatou que tanto a temperatura quanto o molhamento foliar tiveram influência na severidade da doença.

Além dos fatores climáticos, outros fatores também podem ter contribuído para o aumento da doença no campo experimental. De acordo com Jones (1968), o fungo é capaz de sobreviver em restos culturais de soja infectados deixados na superfície do solo. No trabalho desenvolvido por McLean e Roy (1988) foram anotados resultados indicando que seis espécies de plantas daninhas são hospedeiras alternativas com sintomas latentes e são fontes de inóculo potenciais no ciclo da doença causada por C. kikuchii. Deste modo, o aumento do crestamento foliar nas safras de 2006/2007 e 2007/2008 pode ter ocorrido também devido ao aumento das fontes de inóculo primário no campo (Dado não apresentado).

Juliatti et al. (2006), que realizaram dois experimentos em três localidades com diferentes grupos de maturação de soja, informaram que no primeiro experimento $75 \%$ das linhagens foram resistentes; no segundo, $87,5 \%$ das linhagens foram considerados resistentes em todas as repetiçôes. Os autores informaram também que a cultivar padrão Emgopa 313 foi resistente em campo às cercosporioses (Cercospora kikuchii e C. sojina) nas três localidades onde se realizaram os experimentos.

Em conclusão, todos os genótipos avaliados tiveram baixo nível de crestamento foliar e os genótipos com menor incidência da doença foram: GT04-7229, GT04-8987, GT04-8902, GT04-7991, GT04-8218, GT04-9501, GT04-7020, GT04-7505, GT04-7439, GT04-7437 RR, GT04-9192 RR, GT04-8091 RR, GT04-8203, GT049265 RR e GT04-9085 RR.

\section{AGRADECIMENTOS}

Ao Conselho Nacional de Desenvolvimento Científico e Tecnológico - CNPq, e à Coordenaçáo de Aperfeiçoamento de Pessoal de Nível Superior - CAPES, pelo financiamento parcial do estudo e ao Sr. João Luiz Gilioli pelo fornecimento de sementes e pela disponibilidade de área para a realização dos experimentos em Cristalina (GO).

\section{REFERÊNCIAS}

ALMEIDA, A.M.R.; FERREIRA, L.P.; HENNING, A.A.; VELOSO, J.F.S.; YORINORI, J.T. Doenças da Soja. In: KIMATI, H.; AMORIM, L.; BERGAMIN FILHO, A.; CAMARGO, L.E.A.; REZENDE, J.A.M. (Ed.). Manual de Fitopatologia. 3.Ed. São Paulo: Agronomica Ceres, 1997. v.2. p.642-664.

ALMEIDA, A.M.R.; SARTORI, F.; CALVO, E.S.; MARIN, S.R.R.; FUKUJI, T.S. Diferenciação morfo-bio-molecular de isolados de Cercospora kikuchii obtidos de sementes de soja no Brasil. Fitopatologia Brasileira, v.26, p.328. 2001.

ALMEIDA, A.M.R.; PIUGA, F.F.; MARIN, S.R.R.; BINNECK, E.; SARTORI, F.; COSTAMILAN, L.M.; TEIXEIRA, M.R.O.; LOPES, M. Pathogenicity, molecular characterization, and cercosporin content of Brazilian isolates of Cercospora kikuchii. Fitopatologia Brasileira, v.30, p.594-602, 2005. 
EMBRAPA. Recomendaçôes técnicas para a cultura da soja no Paraná 1999/2000. Londrina: Embrapa Soja, 1999. 236p. (Embrapa Soja, Documentos, 131)

EMBRAPA. Tecnologias de Produção de Soja - Região Central do Brasil 2009/2010. Londrina: Embrapa Soja, 2008. 262p. (Sistemas de Produção / Embrapa Soja, n.13)

FEHR, W.R.; CAVINESS, C.E. Stage of soybean development. Ames; Iowa State University, 1981, 12p. (Special report, 80)

GODOY, C.V.; CANTERI, M.G. Efeito da severidade de oídio e crestamento foliar de cercospora na produtividade da cultura da soja. Fitopatologia Brasileira, v.29, p.526-531, 2004.

GUERRA, N.B.; LIVERA, A.V.S. Correlação entre o perfil sensorial e determinaçóes físicas e químicas do abacaxi cv. Pérola. Revista Brasileira de Fruticultura, v. 21, p.32-35, 1999.

HEPPERLY, P.R. Purple seed stain incidence among soybean cultivars and between seasons in Puerto Rico. Journal of Agriculture of the University of Puerto Rico, v.68, p.87-99. 1984.

JONES, J.P. Survival of Cercospora kikuchii on soybean stems in the field. Plant Disease Report, v.52, p.931-934. 1968.

JULIATTI, F.C.; HAMAWAKI, O.T.; CUNHA, E.P.C.; POLIZEL, A.C.; SANTOS, M.A.; SHIGIHARA, D. Severidade de doenças fúngicas foliares em genótipos de soja em três locais de plantio. Bioscience Journal, v.22, p.83-89, 2006.

KLINGELFUSS, L.H.; YORINORI, J.T. Infecção latente de Colletotrichum truncatum e Cercospora kikuchiiem soja. Fitopatologia Brasileira, v.26, p.158-164, 2001.

MARTINS, M.C.; GUERZONI, R.A.; CÂMARA, G.M.S.; MATTIAZZI, P.; LOURENÇO, S.A.; AMORIM, L.. Escala diagramática para a quantificação do complexo de doenças foliares de final de ciclo em soja. Fitopatologia Brasileira. v.29, p.179-184, 2004.

MCLEAN, K.S.; ROY, K.W. Purple seed stain of soybean caused by isolates of Cercospora kikuchii from weeds. Canadian Journal of Plant Pathology, v. 10, p.166-171, 1988.
MILLER, W.A.; ROY, K.W. Effects of benomyl on the colonization of soybean leaves, pods, and seeds by fungi. Plant Disease, v.66, p.918-920, 1982 .

OLIVEIRA, W.F.; CAETANO, F.V.; DIAS, E.M.; BATISTA, R.G.; NONATO, A.R. Eficiência de produtos fitossanitários pulverizados na cultura da soja (Glycine max L.), no controle da mancha parda (Septoria glycines) e crestamento foliar-mancha púrpura dos grãos (Cercospora kikuchii). Pesquisa Agropecuária Tropical, v.30, p.63-66, 2000.

ORTH, C.E.; SHUH, W. Resistance of 17 soybean cultivars to foliar, latent, and seed infection by Cercospora kikuchii. Plant Disease, v.78, p.661-664, 1994.

ROY, K.W.; ABNEY, T.S. Purple seed stain of soybeans. Phytopathology, v.66, p.1045-1049, 1976.

SANTOS, F.S.; SOUZA, P.E.; POZZA, E.A.; MIRANDA, J.C.; CARVALHO, E.A.; FERNANDES, L.H.M.; POZZA, A.A.A. Adubação orgânica, nutrição e progresso de cercosporiose e ferrugem-do-cafeeiro. Pesquisa Agropecuária Brasileira, v.43, p.783-791, 2008.

SCHUH, W. Influence of temperature and leaf wetness period on conidial germination in vitro and infection of Cercospora kikuchii on soybean. Phytopathology, v.81, p.1315-1318, 1991.

SINCLAIR, F.B.; BACKMAN, P.A. (Ed). Compendium of soybean diseases. 3.Ed. St. Paul: American Phytopathological Society, 1989. $106 \mathrm{p}$.

STEEL, R.; TORRIE, J. Principles and procedures of statistics: a biometrical approach. 2.Ed. New York: McGraw-Hill, 1980. 631p.

WALTERS, H.J. Cercospora leaf blight of soybeans. Phytopathology News, v.12, p.165-166, 1978.

WILCOX, J.R.; ABNEY, T.S. Effects of Cercospora kikuchii on soybeans. Phytopathology, v.63, p.796-797, 1973.

WRATHER, J.A.; ANDERSON, T.R.; ARSYAD, D.M.; GAI, J., PLOPER, L.D.; PORTA-PUGLIA, A.; RAM, H.H. Soybean disease loss estimates for the top 10 soybean producing countries in 1994. Plant Disease, v.81, p.107-110, 1997. 